\title{
Stress and Coping in Nurses Taking Care of People Living with HIV in Hunan, China: A Descriptive Qualitative Study
}

\author{
Chen Pan $\mathbb{D}^{\prime}$, Honghong Wang $\mathbb{D}^{2}$, Minzhen Chen $\mathbb{D}^{3}$, Yu Cai $\mathbb{D}^{1}$, Peihuan $\mathrm{Li}^{4}$, Changgen Xiao $\mathbb{D}^{5}$, \\ Qiuping Tang $\mathbb{D}^{1}$, Deborah Koniak-Griffin ${ }^{6}$
}

\begin{abstract}
'Department of Clinical Psychology, The Third Xiangya Hospital of Central South University, Changsha, People's Republic of China; ${ }^{2}$ Department of Fundamental Nursing, Xiangya School of Nursing, Central South University, Changsha, People's Republic of China; ${ }^{3}$ Department of AIDS, The First Hospital of Changsha, Changsha, People's Republic of China; ${ }^{4}$ College of Resources of Environment, Hunan Agricultural University, Changsha, People's Republic of China; ${ }^{5}$ Department of Applied Psychology, Hunan University of Chinese Medicine, Changsha, People's Republic of China; ${ }^{6}$ School of Nursing, University of California Los Angeles, Los Angeles, CA, USA
\end{abstract}

Correspondence: Qiuping Tang, Department of Clinical Psychology, The Third Xiangya Hospital of Central South University, Changsha, People's Republic of China, Tel +86 73I 88618567, Email tt96@sina.com

Background/Purpose: Nurses engaged in the care of people living with HIV (PLWH) are commonly exposed to workplace stress. This study aimed to explore the stress experiences and coping strategies among nurses taking care of PLWH in China.

Methods: Nurses were recruited from the AIDS department of a public, general, third-grade class-A hospital, which has the largest HIV care department in the Hunan Province of China. Thirty-three nurses working in the AIDS Department were recruited in this qualitative study. Eight nurses participated in a focus group and 25 nurses underwent in-depth individual interviews aimed at characterizing the nurse's feelings and struggles with stress during caregiving for PLWH. The interviews were audio-recorded, transcribed verbatim, anonymized, and imported into NVivo 8.0 software. The data were coded and subjected to thematic analysis.

Results: Concerns about occupational exposure, heavy workload, mental health problems and risk behaviors of patients, and discrimination towards nurses caring for PLWH were the four main sources of stress. The negative impact of stress included problems with emotion regulation, somatic health and sleep, and work performance. Some participants also reported a positive impact of work stress on their mental health. Using personality strengths, problem-solving, help-seeking, concealing and avoiding/suppression were common coping strategies employed by nurses caring for PLWH.

Conclusion: Our findings help characterize the stress experienced by nurses caring for PLWH in the Chinese cultural context, and may inform specific interventions to help manage stress and promote mental health of nurses.

Keywords: HIV, nurses, stress, job burnout, coping

\section{Introduction}

Nurses are typically exposed to a high level of occupational stress. ${ }^{1,2}$ The contributing factors include long working hours, shortage of human resources, increasing complexity of medical care, and personal and patient safety concerns. In addition, nurses have to routinely deal with loss, pain, and psychologically distressful situations as part of their work and have to bear the burden of providing emotional support to patients. ${ }^{1,2}$ Workplace stress adversely affects the well-being ${ }^{3}$ and mental health of nurses, and decreases the quality of care. ${ }^{4}$

Shortage of nurses is a global phenomenon that is expected to persist. According to the World Health Organization, nearly half of the countries in the world have reported fewer than three nurses per 1000 population, while $27 \%$ of the countries have reported less than 1 nurse per 1000 population. ${ }^{5}$ A recent study revealed a poor nurse to patient (NTP) ratio in large general hospitals in China (mean NTP $=1: 8.0$ during day shift and 1:23 during night shift). ${ }^{6}$ The enormous population in China and the low NTP aggravates the stress experienced by nurses, placing them at high risk for job burnout. ${ }^{7}$ In a cross-sectional survey conducted in China, the mean Occupational Stress Questionnaire (OSQ) scores of 
nurses were $5 \%-6 \%$ higher than those of professionals in other occupations such as primary and secondary school teachers within the country, indicating that nurses are at a higher risk of occupational stress. ${ }^{8}$ Studies conducted during the ongoing COVID-19 pandemic have shown that frontline nurses are at a greater risk of depression. ${ }^{9,10}$

Studies have shown that among health personnel engaged in the care of people living with HIV (PLWH), nurses experience comparatively higher work-related stress than laboratory staff or peer counselors, ${ }^{11}$ creating a major challenge in the provision of HIV care. Nurses engaged in the care of PLWH may experience stress, burnout, and even leave the profession, especially when physical contact with PLWH elicits misunderstandings, avoidance, and discrimination by the social contacts and family members. ${ }^{12}$ For these nurses, stress and burnout may be exacerbated because of the challenging nature of this patient population; for example, multiple hospitalizations, poor treatment adherence, and fear of dying.

Two previous studies have shown that nurses caring for PLWH in China experience higher stress levels than nurses in other departments. ${ }^{13,14}$ The stress was found largely attributable to the general work-related conditions such as heavy workload, unrealistic work environment and demands, institutional culture including leadership style, relationships with colleagues and leaders, and HIV/AIDS-related stress including complexity of medical conditions, fear of occupational exposure, psycho-social status of patients, and public stigma against nurses working with PLWH. ${ }^{15-17}$ Nurses have to cope with the pressure of work while striving to gain understanding and acceptance from families and society about their professional role. This situation places them at high risk of stress and burnout. ${ }^{15}$ In addition, protective measures against HIV infection are not perfect. ${ }^{18}$ Studies have shown a high prevalence of sharp injuries among Chinese health care workers engaged in AIDS prevention and control (31.8\% versus $0.3 \%-0.5 \%$ in Western countries). ${ }^{19}$ The fear of getting infected because of sharps injuries renders Chinese nurses more vulnerable to experience burnout. ${ }^{19}$ The reported overall prevalence of job burnout syndrome among HIV health care professionals in China is $76.9 \% .^{18}$

Coping strategies play an important role in ameliorating the occupational stress experienced by nurses. In a study, planning, seeking emotional social support, and seeking instrumental social support were found to be the coping strategies applied by general nurses in Poland. ${ }^{20}$ Avoidance strategy, social support, faith-based practices, and wishful thinking were the reported coping strategies applied by nurses during the ongoing COVID-19 pandemic. ${ }^{21,22}$ According to a study conducted in USA, the coping strategies employed by nurses working in AIDS care include balancing personal and professional life, releasing pain, respecting yet controlling feelings, managing demands, and seeking help. $^{23}$

The nature of stress experienced by nurses caring for PLWH in China is not well characterized, and previous studies have mostly applied quantitative research methods. ${ }^{24}$ Use of qualitative research methods can provide more in-depth information by enhancing our understanding of the lived experiences of nurses caring for PLWH that may contribute to their stress and burnout.

\section{Materials and Methods}

\section{Aim}

The aim of this study was to explore the stress experiences among nurses caring for PLWH, including the source of stress, the impact of stress, and the coping strategies employed to deal with the stress.

\section{Study Design}

A descriptive qualitative study was conducted with data collected through a focus group followed by semi-structured individual in-depth interviews. Focus groups offer the advantage of allowing exploration of attitudes within the social setting wherein they are created and exchanged, while individual in-depth interviews help obtain individual perspectives and provide confidentiality to participants. ${ }^{25}$ The focus group was conducted prior to the individual interviews in order to obtain a wide range of information from participants who differed in age, nursing experiences, and communication skills. Thematic framework analysis ${ }^{26}$ was used to analyze the data. Thematic framework analysis is a well-established method for analysis of qualitative data. It ensures the rigor, transparency, and operability of the data processing and analysis process. $^{27}$ 


\section{Setting and Participants}

The study participants were enrolled from the AIDS Department of a public general hospital, which has the largest HIV care department in a South-Central Chinese Province, providing care to approximately 15,000 PLWH per year. A total of 43 nurses provide care to PLWH within the hospital's two inpatient wards, a clinic, and a research center.

A purposive sample of 33 nurses working in the AIDS department was recruited through flyers posted in the wards and clinic of the department. Interested nurses contacted the research assistant (RA) and were screened for eligibility during face-to-face interviews by the principal investigator (PI) or RA. Inclusion criteria were: a) having a license to practice as a nurse in the People's Republic of China; b) being employed as a nurse in the AIDS department of the participating hospital for at least half a year; and c) being fluent in Mandarin. Other types of health care providers (eg doctors, pharmacists, social workers) and nursing supervisors or managers were excluded. All the recruited nurses were eligible and agreed to participate in the study. Only verbal informed consent was requested since this was a study of the lowest risk. This study was approved by the Ethics Committee of the Xiangya School of Nursing, Central South University, and was conducted in accordance with the Declaration of Helsinki.

\section{Procedures}

Focus group and in-depth individual interviews were conducted in a private room of the participating hospital. The room was comfortable, with minimal chance of interruption. The PI conducted the sessions. The PI is a PhD-qualified clinical psychologist with postdoctoral training in School of Nursing, University of California, Los Angeles. The PI has participated in the Xiangya-UCLA Nursing and Psychology HIV Research Program during postdoctoral training and has undergone training in qualitative study and mindfulness intervention. The RA was trained in qualitative research and the study methodology, and assisted the PI with data collection and analysis. The team of researchers included psychiatrists, clinical psychologists, and nursing experts who have been engaged in the field of AIDS-related research for many years.

All participants completed a brief survey to collect demographic data (age, gender, marital status, years of nursing practice, and years of nursing experience in HIV/AIDS care) before the focus group or in-depth interviews. Participants were assigned to either the focus group or an in-depth interview according to registration order; those with registration numbers 1-8 participated in the focus group and 9-33 participated in the in-depth interview. Eight nurses participated in the 60-minute focus group, and 25 nurses received in-depth individual interviews, lasting approximately 40 minutes each.

The focus group and in-depth interviews followed a semi-structured interview format with open-ended questions (Table 1). Items in the guide were developed by the research team. The first draft of the interview guide was designed by the PI based on an extensive review of the literature related to stress and burnout of nurses caring for PLWH, and the focus was to identify the source and influence of stress and the coping strategies employed by nurses caring for PLWH within the Chinese historical and cultural contexts. Second, research team members, including experts in psychology, nursing, and HIV/AIDS reviewed the guide and provided feedback. Third, revisions of the interview guide were made based on that feedback. Finally, the focus group was conducted, and the findings led to minor revisions in the interview guide for use in the individual interviews (see Table 1).

To ensure confidentiality, participants were asked not to discuss the information shared during the focus group with anyone outside of their assigned sessions. Participants were informed of their right to refuse to answer any questions and to withdraw from the study at any time without any negative impact on their work. Participants had the opportunity to review the audio recordings and the right to erase all or some of the information.

The language used in focus group/in-depth individual interviews and the analysis software was Chinese. Findings of data analysis were translated into English at the time of drafting the articles. Deborah Koniak-Griffin, the English native author in the research team, has checked the language quality.

\section{Data Analysis}

The digital recordings of the focus group and in-depth interviews were transcribed verbatim. The transcripts were imported and analyzed with NVivo 8.0, a qualitative data analysis software package. The data analysis included the following steps: a) transcribing and reading data; b) coding interesting features of the data and generating initial codes; c) collating codes into 
Table I Focus Group and in-Depth Interview Guide*

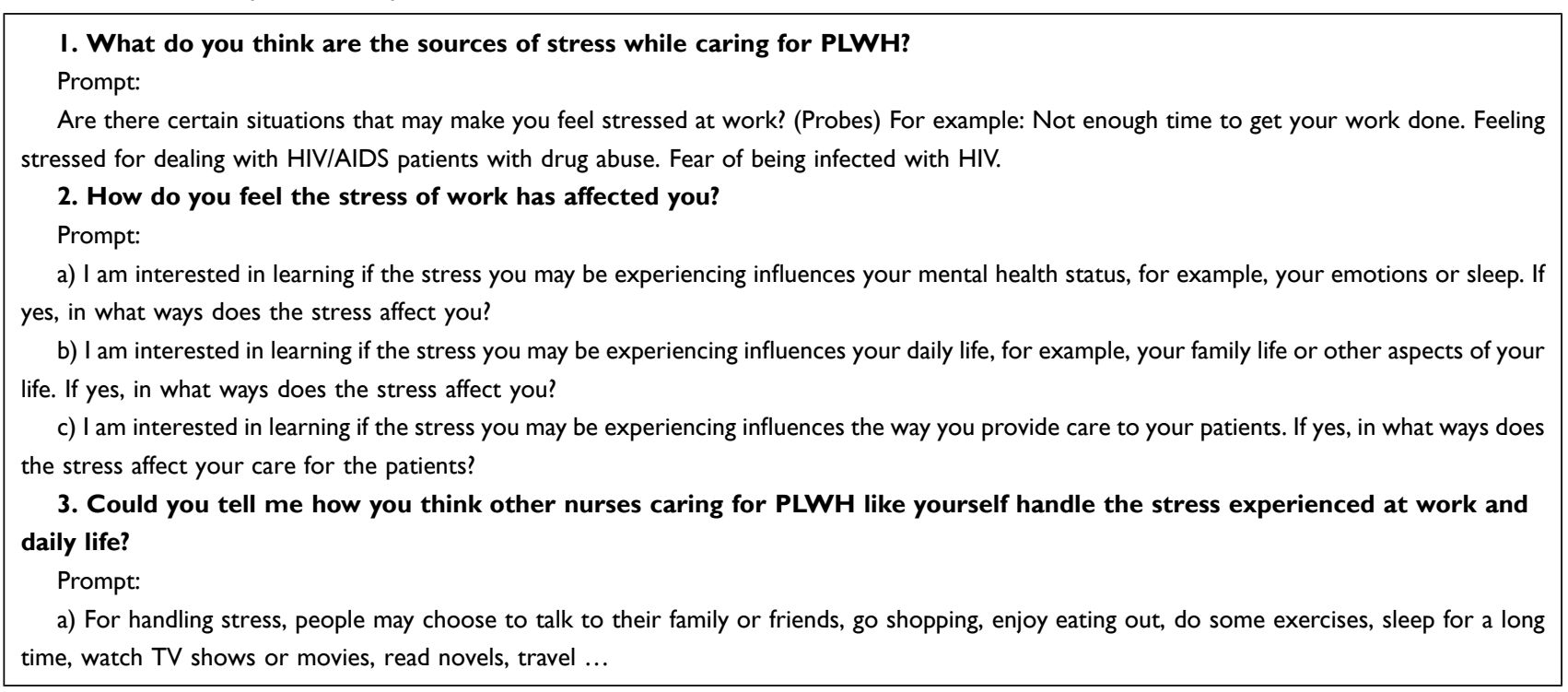

Notes: *Participants receiving individual interviews were asked questions I-3 (above) that were administered in the focus group. In addition, they were asked two related questions: I) What do you think is the most stressful experience at work? 2) Are there any positive effects that you are experiencing from stress? If yes, please explain.

potential themes; d) reviewing themes and generating a thematic map; e) defining and naming themes; f) selecting vivid, compelling extract examples; and g) producing the analysis report. Through data analysis, four subthemes emerged on sources of stress, four subthemes emerged on impact of stress, and two subthemes emerged on coping with stress.

The PI and one RA independently coded the transcripts and compared the individual codes and code clusters. Disagreement, if any, was resolved in consultation with another researcher and a consensus reached. All transcriptions, coding, and analyses were completed in Mandarin, and the results were translated into English for preparing the manuscript. Findings from the focus group and in-depth interviews were combined to report the results.

The demographic characteristics of the study population were analyzed using descriptive statistics with the Statistical Package for the Social Sciences Version 22.0.

\section{Trustworthiness and Credibility}

Several steps were taken to ensure the rigor and credibility of data. First, we invited members of the research team to carefully review the interview guide, and some members of this team were invited to perform role play using the interview guide and subsequently provide feedback. Second, we asked open-ended questions during interviews and used psychological counseling skills to encourage participants to share. Third, the PI and RA were trained in the art of conducting skills prior to the focus group and in-depth interviews. Fourth, in addition to audio recordings, the PI or RA collected observational data and made notes during the focus group and in-depth interviews. For example, notes were made on the length of interviews, interruptions, and non-verbal cues (eg, facial and eye expressions) during the discussions. Fifth, peer checking was used to ensure qualitative reliability and credibility. One RA transcribed the interviews, and after the transcripts were completed, the PI reviewed the data and verified that the transcripts adequately reflected the nurses' experiences. This report followed the guidelines of the consolidated criteria for reporting qualitative studies (COREQ).

\section{Results}

A total of 33 nurses participated in the study, including eight nurses in the focus group and 25 nurses in the in-depth individual interviews. Table 2 summarizes the sociodemographic characteristics of the study population. The mean age of participants was 28.33 years (range, 21-50). The majority of participants $(97 \%)$ were female. The average length of employment in nursing was 7.85 years (range, 2-31), with an average length of 4.44 years' employment caring for PLWH (range, 0.5-13). 
Table 2 Sociodemographic Characteristics of the Participants

\begin{tabular}{|c|c|c|c|}
\hline \multicolumn{2}{|l|}{ Sociodemographic Characteristics } & \multirow{2}{*}{$\frac{n}{1}$} & \multirow{2}{*}{$\begin{array}{l}\% \text { of Total } \\
3.0\end{array}$} \\
\hline Gender & Male & & \\
\hline & Female & 32 & 97.0 \\
\hline \multirow[t]{2}{*}{ Age } & $M \pm S D$ & $28.33 \pm 6.34$ & \\
\hline & range & $21-50$ & \\
\hline \multirow[t]{3}{*}{ Degree } & Associate degree & II & 33.3 \\
\hline & Bachelor degree & 21 & 63.6 \\
\hline & Master degree or doctor degree & I & 3.0 \\
\hline \multirow[t]{4}{*}{ Title } & Junior nurse & 12 & 36.4 \\
\hline & Senior nurse & 14 & 42.4 \\
\hline & Charge Nurse & 6 & 18.2 \\
\hline & Associate chief senior nurse & I & 3.0 \\
\hline \multirow[t]{2}{*}{ Years of nursing practice } & $\mathrm{M} \pm \mathrm{SD}$ & $7.85 \pm 7.05$ & \\
\hline & range & $2-31$ & \\
\hline \multirow[t]{2}{*}{ Years of nursing practice in HIV/AIDS care } & $\mathrm{M} \pm \mathrm{SD}$ & $4.44 \pm 3.363$ & \\
\hline & range & $0.5-13$ & \\
\hline \multirow[t]{5}{*}{ Marital status } & Single & 13 & 39.4 \\
\hline & Married & 17 & 51.5 \\
\hline & Divorced & I & 3.0 \\
\hline & Widowed & I & 3.0 \\
\hline & Refuse to answer & I & 3.0 \\
\hline \multirow[t]{4}{*}{ Have any diseases } & Yes (depression) & 3 & 9.1 \\
\hline & No & 29 & 87.9 \\
\hline & Refuse to answer & I & \\
\hline & & & 3.0 \\
\hline \multirow[t]{3}{*}{ Using any psychotropic medications } & Yes (estazolam) & 2 & 6.1 \\
\hline & No & 29 & 87.9 \\
\hline & Refuse to answer & 2 & 6.1 \\
\hline
\end{tabular}

Abbreviation: M, ; SD, standard deviation.

Through qualitative data analysis, we identified four subthemes related to sources of stress, four subthemes related to the impact of stress, and two subthemes related to coping with stress (Tables 3-4).

\section{Sources of Stress While Working in the HIVIAIDS Department Concern About Occupational Exposure}

Most participants reported that they felt great stress about their risk of contracting HIV infection at work. Nearly half of the participants $(12 / 25,48 \%)$ in the in-depth interviews and participants in focus group considered occupational exposure to be their biggest source of stress (Table 4). Some nurses had experienced occupational exposure by direct contact with blood or bodily fluids of PLWH, despite exercising due precautions when providing care. Most occupational exposures were related to sharp (needle) injuries during injection or drawing blood, and others were related to splashing of body fluid during blood glucose measurements and suction procedures. Nurses experiencing occupational exposure were required to continue working after a few days of rest regardless of any side effects of the drugs used for post-exposure prophylaxis.

In our department, seven cases of occupational exposure occurred this year, including me. We had to take antiviral drugs for 38 consecutive days after occupational exposure. Some people had very severe side effects, including vomiting, and were unable to eat anything. Staff can only take five days off for occupational exposure. We have to work despite suffering very severe side effects from our prophylactic medicine due to shortage of nurses. [P3] 
Table 3 Themes Related to Stress and Coping in Nurses Taking Care of People Living with HIV Identified in the Data Analysis

\begin{tabular}{|l|l|l|l|}
\hline Themes & Sources of Stress & Influences of Stress & Coping Strategies \\
\hline Subthemes & $\begin{array}{l}\text { Concern about occupational } \\
\text { exposure }\end{array}$ & $\begin{array}{l}\text { Dysfunctional emotional } \\
\text { regulation }\end{array}$ & $\begin{array}{l}\text { Positive coping: } \\
\text { Using personality strengths } \\
\text { Using problem-solving skills } \\
\text { Seeking help }\end{array}$ \\
\cline { 2 - 4 } & Heavy workload & $\begin{array}{l}\text { Somatic symptoms and } \\
\text { altered sleep patterns }\end{array}$ & $\begin{array}{l}\text { Negative coping: } \\
\text { Concealing } \\
\text { Avoiding/Suppression }\end{array}$ \\
\cline { 2 - 4 } & $\begin{array}{l}\text { Patients' mental health problems and } \\
\text { aggressive behaviors }\end{array}$ & Affected work quality & \\
\cline { 2 - 4 } & Perceived discrimination & Positive influences & \\
\hline
\end{tabular}

Table 4 Sources, Influence, and Coping Strategies of Stress of Nurses Taking Care of People Living with HIV in Hunan, China

\begin{tabular}{|c|c|c|}
\hline Themes and Subthemes & $\begin{array}{l}\text { Checked By Focus } \\
\text { Group }(n=8)\end{array}$ & $\begin{array}{l}\text { Responses Through In-Depth } \\
\text { Interviews } \\
(\mathbf{n}=\mathbf{2 5})\end{array}$ \\
\hline \multicolumn{3}{|l|}{ Sources of stress } \\
\hline Concern about occupational exposure & Yes & $12(48)$ \\
\hline Heavy workload & Yes & $19(76)$ \\
\hline $\begin{array}{l}\text { Patients' mental health problems and } \\
\text { aggressive behaviors }\end{array}$ & Yes & $17(68)$ \\
\hline Perceived discrimination & No & $14(56)$ \\
\hline \multicolumn{3}{|l|}{ Influences of Stress } \\
\hline Dysfunctional emotional regulation & Yes & $21(84)$ \\
\hline Somatic symptoms & Yes & $8(32)$ \\
\hline Altered sleep patterns & Yes & $18(72)$ \\
\hline Affected work quality & Yes & $14(56)$ \\
\hline Positive influences & No & $13(52)$ \\
\hline \multicolumn{3}{|l|}{ Coping strategies } \\
\hline \multicolumn{3}{|l|}{ Positive coping } \\
\hline Using personality strengths & No & $7(28)$ \\
\hline Using problem-solving skills & Yes & $15(60)$ \\
\hline Seeking help & Yes & $24(96)$ \\
\hline \multicolumn{3}{|l|}{ Negative coping } \\
\hline Concealing & Yes & $8(32)$ \\
\hline $\begin{array}{l}\text { Avoiding } \\
\text { Suppression }\end{array}$ & $\begin{array}{l}\text { No } \\
\text { Yes }\end{array}$ & $7(28)^{a}$ \\
\hline
\end{tabular}

Notes: Data are presented as Frequency (Percentage). ${ }^{\text {a}}$ Responses to avoiding/suppression. 


\section{Heavy Workload}

Many participants $(19 / 25,76 \%)$ in the in-depth interviews and focus group reported that heavy workload was an important source of stress associated with lengthy and intensive work, frequent stressful night shifts, and increased difficulty in administering injections because of patients' drug abuse or prolonged hospitalization (Table 4). High NTP ratio and severe nursing shortages are particular problems in the area of HIV care. Patients who are seriously ill with AIDS require multiple treatments and extensive care, which increases the workload of nurses.

We feel very stressed because there are too many patients in the ward and it is crowded, especially in the peak season. In this case, one nurse has to take care of 12-16 patients at the same time. Providing treatment and daily care are all nurses' tasks. [P22]

Each nurse usually has three night shifts per week, which means that half of the week we cannot be with our families. [P3]

\section{Mental Health Problems of Patients and Their Aggressive Behaviors}

Many participants $(17 / 25,68 \%)$ in the in-depth interviews and focus group reported that a great deal of stress experienced by them was attributable to the mental health problems of their patients (Table 4). Patients sometimes have difficulty accepting that they are infected with HIV, and their mental health is usually affected by the pain caused by their illness. Gradually, they develop mood and behavioral changes. Denial, irritability, aggressive behaviors, communication difficulties, and drug abuse are common in this group.

The most important thing is the mental health status of the patient. They can't accept that they have been infected with HIV so sometimes they will demonstrate aggressive behaviors. High medical costs may also make them frustrated. The mood of patient will frequently change and they will not cooperate with treatment. [P17]

Patients are difficult to manage, especially homeless, drug users, and those abandoned by their family or with violent tendencies. We are worried about being hurt by patients with aggressive behaviors. We are also concerned with patients attempting suicide because one patient jumped off a building in the past. [P10]

\section{Perceived Discrimination}

More than half of the participants $(14 / 25,56 \%)$ in the in-depth interviews reported that they were hurt by discrimination from families, colleagues, friends, and other acquaintances (Table 4). Many people, including health professionals, have misconceptions about HIV/AIDS. For example, some people believe that HIV can be transmitted by shaking hands. Discrimination against nurses caring for PLWH is a common phenomenon because of their perceived risk of getting infected. Discrimination is shown by words and non-verbal cues, which usually make nurses feel frustrated and isolated.

Once I went to the Provincial Centers for Disease Control, I handed the file to the staff. He said with a look of disgust, 'Just leave it there,' and he moved his body backwards. When I travel and tell others about working in the AIDS department, their first response is to stay away from me. [P5]

\section{Influences of Stress}

\section{Dysfunctional Emotional Regulation}

Most participants $(21 / 25,84 \%)$ in the in-depth interviews and focus group reported that stress has a negative impact on their emotional regulation and induces mood swings (Table 4). Participants described how they always feel unhappy, anxious, and easily lose their temper for trivial issues. Their families often become scapegoats of their negative emotions, which adversely affects their communication with the family members. Participants' fears usually emanate from worries about becoming infected with HIV.

I am obviously unhappy at work, and even patients can recognize it. I feel depressed about my current job. I don't want to talk to anybody and feel lonely. When I talk to colleagues or patients, I will make them unhappy with my verbal and emotional attacks. [P5]

I often worry about how I will deal with the heavy work the next day. This worry affects my mood at home and my ability to watch TV or do other relaxing activities. [P16] 


\section{Somatic Symptoms and Altered Sleep Patterns}

Some participants $(8 / 25,32 \%)$ in the in-depth interviews and participants in focus group reported a negative effect of work-related stress on their somatic health (Table 4). The effect of mental state on the physical well-being is well documented. Stress can adversely affect both mental health and physical health, and sometimes stress may induce somatic symptoms, such as fatigue, headache, emotional lability, and shortness of breath. Eighteen participants in the in-depth interviews $(18 / 25,72 \%)$ and participants in the focus group discussion claimed that the stress was adversely affecting their sleep, including difficulty in falling asleep in the evening and poor sleep quality (Table 4).

I always feel tired after work. When I go home after work, I usually lie on the couch or bed and do not do any chores, including cooking. [P10]

\section{Affect on Work Quality}

More than half of the participants $(14 / 25,56 \%)$ in the in-depth interviews reported that stress caused communication problems with patients and/or colleagues due to poor concentration, contributing to unsafe clinical nursing practices and diminishing their passion for work (Table 4). They did not like to talk with patients or colleagues, lacked patience when communicating with others, and easily got into quarrels. Participants in the focus group reported that stress interfered with their ability to concentrate (Table 4).

Stress can affect my attitude toward the patient. I become impatient, do not like to have too much contact with patients, and do not want to talk to them. When patients or their family members do not understand me, I have no way to control my emotions and will easily lose my temper with them. [P1]

\section{Positive Influences}

Half of the participants $(13 / 25,52 \%)$ in the in-depth interviews reported that stress also had positive effects, including increasing motivation and working ability, increased understanding of the human mind, and having a sense of satisfaction and achievement (Table 4).

I think that stress can help me face problems more positively; that is, if the pressure is greater, the motivation will be greater. For example, if the patients do not understand me, their mood is fluctuating, and they even want to commit suicide by jumping off the building, I will try my best to enlighten them, ask them to face the disease bravely, and go for psychological counseling. I also attended psychology classes and trainings. I want to understand my own mind and also want to understand other people's minds. [P14]

Some patients were expected to die within a few days; however, my effort helped them to live, and I have a feeling of satisfaction. [P11]

The stress from work usually pushes me to move forward, overcome the difficulties, and improve myself in many aspects. [P4]

\section{Coping Strategies \\ Positive Coping \\ Using Personality Strengths}

Some participants $(7 / 25,28 \%)$ in the in-depth interviews reported that their personality traits helped them to deal with stress effectively (Table 4). Personality traits play an important role in coping with stress, and some participants seemed to have better resilience than others when dealing with stress.

I am good at regulating and counseling myself, so I know how to respond to the pressure. [P22]

\section{Using Problem-Solving Skills}

Many participants $(15 / 25,60 \%)$ in the in-depth interviews reported that when exposed to stress, they took active measures including improving communication skills with patients, enhancing safety protection in nursing care, putting more effort to get the job done well, separating life from work, concentrating on work, and providing health education for 
family and friends. Participants in the focus group reported that they took actions including improving communication skills with patients, enhancing safety protection in nursing care, separating life from work, and concentrating on work (Table 4).

When I communicate with patients, I pay special attention to my words so the patient will feel that I really care about him. I chat more with patients and let them feel my warmth. I have a patient who said that he especially liked my care because I asked how he felt today and whether he felt better. I asked if he slept well and whether he felt better. [P12]

When my friends talk about AIDS, they believe it is a deadly disease, but I tell them that even if we experience occupational exposure, we can take medicine, and the prevention rate is around $99 \%$. In this way, they will know that AIDS is not so horrible. [P12]

\section{Seeking Help}

Most participants $(24 / 25,96 \%)$ in the in-depth interviews reported that when they experienced stress, they sought help or guidance from other people, such as colleagues, supervisors, security guards in the hospital, friends or families, and they engaged in activities for leisure and entertainment. Participants in the focus group reported they took actions such as seeking help from colleagues, supervisors, and engaging in activities for leisure and entertainment (Table 4).

The first reaction to unhappy things at work is to call a colleague because she will understand me better. For example, once my colleague and I were mistreated at work, we cried and confided in each other, and the pressure was released a lot. [P10]

\section{Negative Coping Concealing}

Some participants $(8 / 25,32 \%)$ in the in-depth interviews and participants in the focus group reported that when they faced stress, they took actions including concealing their employment in an HIV department from strangers, relatives, or friends (Table 4).

I used to tell others that I work in the HIV department, but now I don't want to say anything because people look strangely at you. If someone asks me what department I am in, I will give a random answer, concealing the reality of being in the HIV department. [P21]

\section{Avoiding/Suppression}

Some participants $(7 / 25,28 \%)$ in the in-depth interviews stated that when they faced stress, they tried to avoid communication with patients or suppress their emotions. Participants in the focus group reported that they tried to suppress their emotions (Table 4). Participants tried to avoid or maintain a distance from patients who were difficult to get along with, especially those who tended to show aggressive behavior. Participants controlled their temper and emotions when they were irritated by patients' words and behaviors, and usually suppressed their feelings of stress, so it was hard for them to share with others.

In communications, if I find that some patients are difficult to get along with, I try to decrease contact with them. At the same time, I try to improve communications with them by talking in humorous ways. But if the patients' attitude does not change, I also keep distance from them in communication. [P13]

\section{Discussion}

This study identified the sources of stress and the influence of stress experienced by nurses providing AIDS care, and the coping strategies used by them to deal with this stress. Our findings facilitate understanding about the stressful situations encountered by nurses caring for PLWH in a Chinese cultural context and can help inform appropriate interventions to ameliorate their stress. 
This study explored the coping methods employed by nurses caring for PLWH in China using qualitative research methods. Our findings show that nurses employ both positive and negative coping strategies to deal with stress. Positive coping involved using personality strengths, problem solving, and seeking help. Negative coping methods included concealing and avoiding/suppression. Problem-solving and help-seeking were the most common coping methods. These strategies were consistent with those identified in a very recent study of stress coping strategies among HIV/AIDS healthcare providers in China; six general coping strategies identified were: seeking social support, applying problemsolving strategies, adopting healthy lifestyle, developing self-compassion, using mindfulness-based stress reduction methods, and avoidance and escaping. ${ }^{28}$ Coping style, as an intermediary mechanism between stress and health, may have a protective effect on physical and mental health. ${ }^{29}$ In a study conducted by Folkman, positive coping styles were found to reduce psychological stress caused by life events, while poor coping styles contributed to mental health problems such as depression and anxiety. ${ }^{30}$ Therefore, future studies should evaluate training designed to help nurses caring for PLWH effectively deal with stress.

Our study indicates several adverse effects of work-related stress, including problems in emotion regulation, sleep, somatic health, work quality, and relationship with family members. This finding is consistent with previous studies that showed a substantial impact of work-related stress on health care providers working with HIV/AIDS or other diseases, affecting their emotional stability, ${ }^{3}$ individual well-being (digestive disorders, cardiovascular disorders, sleep disorders, and poor immunity function), family and social life, ${ }^{16,31,32}$ and quality of care. ${ }^{16}$ The health status of health care workers has been found to have a moderate direct positive effect on the quality of health care. ${ }^{33}$ Protection motivation theory (PMT) suggests that when health professionals are in good health, they usually provide better health care for patients, especially if they receive service health education and understand health-related behaviors. ${ }^{34}$ Hence, hospital administrators and policy makers should take cognizance of and provide support to promote physical/mental health of nurses caring for PLWH. At the same time, this study found that work stress may have a positive effect on some nurses caring for PLWH by increasing their motivation and working ability, understanding of the human mind, and conferring a sense of satisfaction and achievement. Similar results was reported by a study of 2426 health care workers across 74 hospitals in China, which showed that challenge stress, as measured by number of work assignments, amount of time working, accomplishments, responsibilities, and time pressures, has a direct inverse effect on the physical/mental health of healthcare workers and significant direct positive effect on the quality of health care. ${ }^{33}$ Challenge stress stimulates desirable emotions and encourages people to solve problems in a positive way, and people usually choose to challenge themselves in order to fulfill their desire for self-development, which may induce a positive effect on nurses experiencing high work stress. ${ }^{35}$

Consistent with earlier research, discrimination was found to be an important stressor for nurses caring for PLWH in China. Similar findings have been reported in studies conducted in developing countries such as South Africa and China. This suggests that stigma associated with caring for PLWH is a significant occupational stress for nurses. ${ }^{15,16,36,37}$ Nurses who care for PLWH tend to be discriminated against by society and even by colleagues, friends, and family. ${ }^{16,31}$ However, similar findings are generally not reported in studies conducted on nurses caring for PLWH in developed countries. In Chinese culture, due to the low rate of HIV-related knowledge, there are widespread misconceptions about the condition, especially among people who do not work in the medical field. ${ }^{15}$ People are often afraid of contracting what they perceive as a deadly virus due to physical contact with someone who has HIV. ${ }^{15}$ Therefore, they may choose to avoid contact with PLWH or health professionals caring for them. Furthermore, knowledge about the policies, procedures, universal precautions, and post-exposure prophylaxis in medical settings is reportedly insufficient in China. ${ }^{16}$ Many factors contribute to stigma and discrimination in health care settings, including lack of knowledge about HIV/ AIDS and universal precautions, ${ }^{38,39}$ fear of transmission, ${ }^{39}$ and viewing HIV/AIDS as a consequence of immoral or illegal behaviors such as engaging in sex work, promiscuity, homosexuality, or drug use. ${ }^{39}$

Occupational exposure from needle injuries was the most important source of stress identified in our findings, which is consistent with previous studies of HIV health care providers in China. ${ }^{16}$ The most common mode of occupational exposure among our participants was needle-related sharp injuries while caring for patients with HIV/AIDS. Needlerelated sharp injuries is the most frequently reported cause of occupational exposure in China. ${ }^{40}$ Nurses reportedly 
represent the most vulnerable population for experiencing sharp injuries among healthcare workers employed in hospitals in China. ${ }^{41}$

Several recommendations are made based upon our findings. Particularly important is the provision of additional human, material, and financial resources to the government-owned public hospitals serving PLWH in China. These resources would help improve the quality of nursing care for PLWH. Improvement in the NTP ratio will potentially decrease job burnout and stress. Additional training should be provided to prevent job discrimination and occupational injuries. $^{42}$ Policy-level interventions and allocation of additional resources for nurses caring for PLWH and targeted interventions for decreasing stigma and discrimination directed towards PLWH and their caregivers are key imperatives. Health education interventions and mass media should be leveraged to promote accurate understanding of HIV/AIDS, and raise awareness about the important role and contributions of nurses caring for PLWH within and outside the clinical settings. Continuing education of personnel working in HIV/AIDS department is important to decrease the risk of occupational exposure from needle-related sharp injuries. Hospital administrators should provide more support and care for PLWH. Moreover, they should develop stress management programs and mental health promotion interventions for nurses caring for PLWH. Group activities among colleagues may be planned to promote understanding and communication between colleagues, so that nurses have the opportunity to share their stress and anxiety and receive emotional support as well as learn from each other.

The findings of this study should be considered in relation to the limitation. The PI is a clinical psychologist who has no clinical experience in the AIDS department; this may have influenced the depth of interview. To address this limitation, in future research, we recommend that the interviewer should work in the AIDS department for a period of time before the initiation of the research, so as to obtain an emic perspective of the experience and feelings of nurses in this department.

This study also has several strengths. The combination of a focus group and in-depth interviews enhances our understanding of the participants' experiences. The focus group was conducted prior to the individual interviews in order to capture the entire spectrum of experiences of nursing caring for PLWH. The in-depth interviews then built upon and expanded the initial findings identified in the focus group because participants tend to share more of their personal ideas, thoughts, and experiences in private interview. In general, most of the findings of the focus group and in-depth interviews were consistent and supported each other. However, the private interviews provided more in-depth insights than the focus group. Discrimination as a source of stress, the positive influence of stress, and personality traits as a means of coping were only discussed in the in-depth interviews. Their statements were richer and more vivid and detailed than in the focus group.

\section{Strengths and Limitations of This Study}

This is one of the few qualitative studies examining stress experienced by nurses caring for PLWH in China. The findings help us to understand the sources and influences of stress, and coping strategies among nurses caring for PLWH in the Chinese cultural context.

To our knowledge, this is the first study that employed both focus group and in-depth interview methods to characterize the stress experienced by nurses caring for PLWH in China.

The principal investigator (PI) is a clinical psychologist who has no clinical experience in the AIDS department. This may have influenced the depth of interview.

\section{Conclusion}

Concerns about occupational exposure to HIV, heavy workload, patients' mental health problems and risky behaviors, and discrimination towards nurses caring for PLWH were the four main sources of stress reported. The negative effects of stress on nurses providing care to PLWH included problems with emotional regulation, sleep, somatic health, work performance, and relationships with family and colleagues. Common coping strategies employed by nurses caring for patients with HIV included concealing feelings, avoiding communication with patients, and suppressing emotions. Work stress was found to also have a positive impact on interpersonal communication, problem-solving, and help-seeking. Our 
findings may be useful to policy makers and nursing managers for developing culturally appropriate strategies for stress management and mental health promotion for nurses caring for PLWH.

\section{Abbreviations}

PLWH, People Living with HIV; RA, Research Assistant; PI, Principal Investigator; NTP Ratio, Nurse to Patient; OSQ, Occupational Stress Questionnaire; COREQ, Consolidated Criteria for Reporting Qualitative Studies; PMT, Protection Motivation Theory .

\section{Data Sharing Statement}

The datasets generated and analyzed during the present study are available from the corresponding author on reasonable request.

\section{Ethics Approval and Informed Consent}

This study has been approved by the Ethics Committee of the Xiangya School of Nursing, Central South University (2017 Ethics Approval File No. 2017023). Verbal informed consent was obtained from the nurses at the time of recruitment.

\section{Consent for Publication}

All data published here are under the consent for publication.

\section{Funding}

This research was supported by the Natural Science Foundation of Hunan Province (grant no. 2020JJ4845), Changsha Science and Technology Bureau (grant no. kq1901127), and NIH-FIC Fogarty International Center (grant \# D43 TW009579). The study sponsors did not participate in any study activity including study design; data collection, management, analysis, and interpretation; report writing; the decision to submit the report for publication; and will have no ultimate authority over any of these activities.

\section{Disclosure}

The authors report no conflicts of interest for this work.

\section{References}

1. Chesak SS, Cutshall SM, Bowe CL, Montanari KM, Bhagra A. Stress management interventions for nurses: critical literature review. J Holis Nurs. 2019;37(3):288-295. doi:10.1177/0898010119842693

2. Cohen-Katz J, Wiley SD, Capuano T, Baker DM, Kimmel S, Shapiro S. The effects of mindfulness-based stress reduction on nurse stress and burnout, Part II: a quantitative and qualitative study. Holist Nurs Pract. 2005;19(1):26-35.

3. Gelsema TI, van der Doef M, Maes S, Janssen M, Akerboom S, Verhoeven C. A longitudinal study of job stress in the nursing profession: causes and consequences. J Nurs Manag. 2006;14(4):289-299.

4. O’Brien-Pallas L, Shamian J, Thomson D, et al. Work-related disability in Canadian nurses. J Nurs Scholarsh. 2004;36(4):352-357.

5. Organization WH. Density of nursing and midwifery personnel (total number per 1000 population, latest available year). Available from: http:// www.who.int/gho/health_workforce/nursing_midwifery_density/en/. Accessed January 28, 2022. 2017.

6. Shen Y, Jian W, Zhu Q, Li W, Shang W, Yao L. Nurse staffing in large general hospitals in China: an observational study. Hum Resour Health. 2020;18(1):3.

7. Ding Y, Yang Y, Yang X, et al. The mediating role of coping style in the relationship between psychological capital and burnout among Chinese nurses. PLoS One. 2015;10(4):e0122128.

8. Wu H, Chi TS, Chen L, Wang L, Jin YP. Occupational stress among hospital nurses: cross-sectional survey. J Adv Nurs. 2010;66(3):627-634

9. Pasam T, Pasam C, Dake R, Soren DK. Incidence of depression, anxiety and sleep disorders in healthcare personal after the onset of Covid 19 pandemic - a survey based study. Crit Care Innov. 2021;4:1-10.

10. Chen X, Liu P, Lei GF, Tong L, Wang H, Zhang XQ. Sleep quality and the depression-anxiety-stress state of frontline nurses who perform nucleic acid sample collection during covid-19: a cross-sectional study. Psychol Res Behav Manag. 2021;14:1889-1900.

11. Bhembe LQ, Tsai FJ. Occupational stress and burnout among health care workers caring for people living with hiv in Eswatini. $J$ Assoc Nurses AIDS Care. 2019;30(6):639-647.

12. Hamama L, Tartakovsky E, Eroshina K, et al. Nurses' job satisfaction and attitudes towards people living with HIV/AIDS in Russia. Int Nurs Rev. 2014;61(1):131-139.

13. Chen M, Pan C, Yang L. Correlation between work pressure and subject well-being in nurses of AIDS wards. Pract Prev Med. 2019;26:542-545.

14. Tang G. Investigation and analysis of working stress and social support of nurses in AIDS ward. Nurs Pract Res. 2009;23:116-117. 
15. Mo Y. Related factors and countermeasures for nurse burnout in AIDS ward. J Univ South China (Medical Edition). $2010 ; 38: 437-439$.

16. Tong H, Li X, Qiao S, et al. Sources and impact of work-related stress among hiv/aids health care providers in guangxi, China: a qualitative research. Workplace Health Saf. 2020;68(2):81-91.

17. Xu G, Ni R, Wu J, Luo J, Lu S. Qualitative study on the working stress of nurses in AIDS ward. Mord Clin Nurs. 2008;7:19-22.

18. Qiao Z, Chen L, Chen M, et al. Prevalence and factors associated with occupational burnout among HIV/AIDS healthcare workers in China: a cross-sectional study. BMC Public Health. 2016;16:335.

19. Li X. Occupational protection aimed at AIDS among medical staff in Operating room. Chin J Gene Pract. 2013;11:275-276.

20. Betke K, Basińska MA, Andruszkiewicz A. Sense of coherence and strategies for coping with stress among nurses. BMC Nurs. $2021 ; 20(1): 107$.

21. Kotrotsiou S, Theofanidis D, Malliarou M, et al. Investigating nurses stress response strategies during the covid-19 pandemic. Mater Sociomed. 2021;33(3):168-173.

22. Sehularo LA, Molato BJ, Mokgaola IO, Gause G. Coping strategies used by nurses during the COVID-19 pandemic: a narrative literature review. Health SA. 2021;26:1652.

23. Sherman DW. Experiences of AIDS-dedicated nurses in alleviating the stress of AIDS caregiving. J Adv Nurs. $2000 ; 31(6): 1501-1508$.

24. Chen J, Cao X, Wu Z. Research progress in job burnout among HIV-related health care workers. Chin J Epidemiol. $2015 ; 36(9): 1020-1022$.

25. Lynch L, Long M, Moorhead A. Young men, help-seeking, and mental health services: exploring barriers and solutions. Am $J$ Mens Health. 2018;12(1):138-149.

26. Braun V, Clarke V. Using thematic analysis in psychology. Qual Res Psychol. 2006;3:77-101.

27. Wang T, Chen J, Hu D. Qualitative data analysis using thematic framework method. Health Res China. 2006;9:86-88.

28. Tong H, Zhou Y, Li X, et al. Stress coping strategies and their perceived effectiveness among HIV/AIDS healthcare providers in China: a qualitative study. Psychol Health Med. 2021:1-11.

29. Li Y, Tang L, Mei G. Work stressors and coping styles in nurses with master's degree in nursing. J Nanchang Univ. 2019;59:90-93.

30. Folkman S, Lazarus RS, Gruen RJ, DeLongis A. Appraisal, coping, health status, and psychological symptoms. J Pers Soc Psychol. 1986;50 (3):571-579.

31. van Dyk AC. Occupational stress experienced by caregivers working in the HIV/AIDS field in South Africa. Afr J AIDS Res. 2007;6(1):49-66.

32. Sun P, Zhang X, Sun Y, et al. Workplace violence against health care workers in north Chinese hospitals: a cross-sectional survey. Int J Environ Res Public Health. 2017;14:1.

33. Ma T, Yang T, Guo Y, Wang Y, Deng J. Do challenge stress and hindrance stress affect quality of health care? Empirical evidence from China. Int J Environ Res Public Health. 2018;15:8.

34. Milne S, Sheeran P, Orbell S. Prediction and intervention in health-related behavior: a meta-analytic review of protection motivation theory. $J$ Appl Soc Psychol. 2000;30:106-143.

35. Yang T, Ma M, Zhu M, et al. Challenge or hindrance: does job stress affect presenteeism among Chinese healthcare workers? J Occup Health. 2018;60(2):163-171.

36. Roomaney R, Steenkamp J, Kagee A. Predictors of burnout among HIV nurses in the Western Cape. Curationis. $2017 ; 40(1)$ :e1-e9.

37. Haber DB, Roby JL, High-George LD. Stigma by association: the effects of caring for HIV/AIDS patients in South Africa. Health Soc Care Community. 2011;19(5):541-549.

38. Pisal H, Sutar S, Sastry J, et al. Nurses' health education program in India increases HIV knowledge and reduces fear. J Assoc Nurses AIDS Care. 2007;18(6):32-43.

39. Sengupta S, Strauss RP, Miles MS, Roman-Isler M, Banks B, Corbie-Smith G. A conceptual model exploring the relationship between HIV stigma and implementing HIV clinical trials in rural communities of North Carolina. N C Med J. 2010;71(2):113-122.

40. Cheng MR, Teng SP, Hua CY, Li BX, Liu YH, Wu PY. Experience of occupational exposure and protection of nurses. Chin J Misdiagnostics. $2011 ; 11: 335-336$.

41. Huang SL, Lu Q, Fan SH, et al. Sharp instrument injuries among hospital healthcare workers in mainland China: a cross-sectional study. BMJ Open. 2017;7(9):e017761.

42. Sun D. Reforming government management system for public hospitals, the only way out for the reform of public hospitals. China Health Law. 2019;27:82-86.

\section{Publish your work in this journal}

Neuropsychiatric Disease and Treatment is an international, peer-reviewed journal of clinical therapeutics and pharmacology focusing on concise rapid reporting of clinical or pre-clinical studies on a range of neuropsychiatric and neurological disorders. This journal is indexed on PubMed Central, the 'PsycINFO' database and CAS, and is the official journal of The International Neuropsychiatric Association (INA). The manuscript management system is completely online and includes a very quick and fair peer-review system, which is all easy to use. Visit http://www.dovepress.com/testimonials.php to read real quotes from published authors.

Submit your manuscript here: https:/www.dovepress.com/neuropsychiatric-disease-and-treatment-journal 